\title{
The Efficacy and Safety of Longmu Tang Granule for Treating Atopic Dermatitis: Study Protocol for a Single-Centered, Double-Blinded, Randomized Controlled Trial
}

\section{YAQIN LI}

Xiyuan Hospital of China Academy of Chinese Medical Sciences

\section{TAOTAO SHEN}

Xiyuan Hospital of China Academy of Chinese Medical Sciences

\section{QINGYING WANG}

Xiyuan Hospital of China Academy of Chinese Medical Sciences

\section{MENGXI MA}

Xiyuan Hospital of China Academy of Chinese Medical Sciences

\section{FENGYAN TIAN}

Xiyuan Hospital of China Academy of Chinese Medical Sciences

\section{YUANYAO SHE}

Xiyuan Hospital of China Academy of Chinese Medical Sciences

\section{YICHENG TAO}

Xiyuan Hospital of China Academy of Chinese Medical Sciences

\section{JINGJING WANG}

Xiyuan Hospital of China Academy of Chinese Medical Sciences

\section{HUIYAN CHI}

Xiyuan Hospital of China Academy of Chinese Medical Sciences

\section{NA LANG ( $\square$ langna96@126.com)}

Xiyuan Hospital of China Academy of Chinese Medical Sciences https://orcid.org/0000-0001-58373641

\section{JIANXUN REN}

Xiyuan Hospital of China Academy of Chinese Medical Sciences

\section{Study protocol}

Keywords: Atopic dermatitis, anti-inflammation, Longmu Tang granule, Randomized controlled trial, Traditional Chinese medicine 
DOI: https://doi.org/10.21203/rs.3.rs-800853/v1

License: (c) (1) This work is licensed under a Creative Commons Attribution 4.0 International License. Read Full License 


\section{Abstract}

Background: Atopic dermatitis (AD) is a chronic relapsing skin disease that has long-term adverse effects on the physical and mental health of children. Current treatments mainly include anti-inflammatory, antibacterial, and anti-allergic interventions, systemic therapy and recently emerging targeted agents. However, these treatments have limited effectiveness and bothersome side effects. The use of traditional Chinese medicine (TCM) is associated with safety and improvements in the clinical symptoms and quality of life of $A D$ patients. However, the poor quality and heterogeneity of studies have not led to sufficient conclusions. Therefore, we will conduct a randomized, controlled, double-blind clinical trial to confirm the efficacy and safety of Longmu Tang granule for the treatment of AD.

Methods: This is a single-center, double-blind, randomized, controlled clinical trial. A total of 60 participants will be randomly assigned (1:1) to receive Longmu Tang granule or placebo granule for 8 weeks. The primary outcome is the index of Scoring Atopic Dermatitis (SCORAD). The second outcomes include the children's Dermatology Life Quality Index (CDLQI) and the Number Cancel Test (NCT), and long-term efficacy. Mechanistic outcome is the serum level of inflammatory cytokines, including IgE, tumor necrosis factor (TNF) -a, interleukin-1 (IL-1) and interleukin-6 (IL-6).

Discussion: The results of this trial will provide evidence on the efficacy and safety of Longmu Tang granule and prove anti-inflammation of Longmu Tang granule for patients with AD.

Trial registration: Chinese Clinical Trial Registry Chictr.org ID: ChiCTR2100041591. Registered on 1 January 2021. https://www.chictr.org.cn/showproj.aspx?proj=62586

\section{Administrative Information}

Trials guidance: please include this text in your protocol just above the Administrative information table:

\section{Note}

the numbers in curly brackets in this protocol refer to SPIRIT checklist item numbers. The order of the items has been modified to group similar items (see http://www.equator-network.org/reportingguidelines/spirit-2013-statement-defining-standard-protocol-items-for-clinical-trials/). 
Title $\{1\}$

A double-blinded, placebo-controlled randomized trial evaluating the efficacy and safety of Longmu Tang granule for treating atopic dermatitis associated with damp-heat disturbing heart-mind: study protocol for a randomized controlled trial.

Trial registration Chictr.org ID: ChiCTR2100041591. Registered on 1 January 2021.

$\{2 a$ and $2 b\}$.

Protocol version 14, May 2020, V3.2

$\{3\}$

Funding $\{4\} \quad$ Supported by Chinese Capital's Funds for Health Improvement and Research (No.2020-2-4172-49)

Author details Department of Dermatology, Xiyuan Hospital of China Academy of Chinese \{5a\} Medical Sciences, Beijing 100091, China

Name and Contact number: 8610-88549772

contact

information for

the trial sponsor

$\{5 b\}$

Role of sponsor

$\{5 c\}$

Capital's Funds for Health Improvement and Research will provide the funding for this trial and supervise its progress

\section{Introduction}

\section{Background and rationale $\{6 a\}$}

Atopic dermatitis (AD) is one of the most common, chronic relapsing skin diseases and is characterized by severe itching, papules, dryness, exudation, and scarring in infants, children, or adults ${ }^{[1]}$. A recent epidemiologic study showed that the prevalence of $A D$ was as high as $10 \%$ to $20 \%$ in developed countries ${ }^{[2]}$. AD affects up to $12.94 \%$ of children aged 1 to 7 years in 12 cities in China ${ }^{[3]}$. $A D$ is not only associated with food allergies, asthma, allergic rhinitis, and other immune-mediated inflammatory diseases, but recent literature suggests it is also associated with attention-deficit/hyperactivity disorder $(A D H D)$ and mental health disorders such as depression ${ }^{[4-7]}$. The typically clinical features of $A D$ are intensive pruritic and eczematous dermatitis which can severely disturb the quality of life (QoL). It's found that $A D$ has a greater impact on children's quality of life than other chronic childhood diseases, including asthma and type 1 diabetes ${ }^{[8]}$. Families and caregivers experienced significant stress and high expenses due to difficult and time-consuming treatments ${ }^{[9]}$. Thus, AD is associated with substantial distress, reduced quality of life and high time and resource demanding for families.

Although the underlying pathogenesis of $A D$ is not fully understood, it is known to be caused by the complex interactions of abnormal barrier function, cutaneous microbiome invasion, and a predominantly type-2-skewed immune dysregulation ${ }^{[10]}$. AD lesions show destruction of the skin barrier caused by inflammatory cytokines from T helper (Th) 2 cell-mediated immunity. Patients with mild-to-moderate AD develop red, oozing, crusted rashes, which have chronic recurrence and may be limited to the face, scalp, 
hands, arms, feet or legs. Pruritus is the major subjective symptom and causes frequent scratching, which may exacerbate the itch-and-scratch-cycle and result in secondary skin infections. Pruritus and frequent scratching at night cause difficulty in falling asleep and impact the quality of life. The lack of sleep at night and early waking can induce adverse consequences on children suffering $A D$, such as psychological disorders, inattention, behaviour changes, and difficulty in remembering daily affairs. Thus, it is necessary for novel therapeutic strategies to alleviate chronic inflammation, including antibacterial and anti-allergic effects, reduce chronic itch and improve the quality of life in the long-term management of $A D$.

Traditional Chinese medicine (TCM) has shown multitargeted effectiveness and safety in improving symptom severity and quality of life of $A D$ patients, especially for patients with refractory $A D{ }^{[11,12]}$. According to TCM theory, damp-heat disturbing heart-mind is the most common symptom in the pathogenesis of $A D$, characterized by fatigue, shortness of breath, spontaneous perspiration or night sweating palpitation, dry mouth, attention deficits, extroversion, restlessness, insomnia and other symptoms. Longmu Tang is composed of Os Draconis (Long Gu) $30 \mathrm{~g} / 126 \mathrm{~g}$, Concha Ostreae (Calcined Mu Li) $30 \mathrm{~g} /$ 126g, Forsythia suspensa (Lian Qiao) $15 \mathrm{~g} / 126 \mathrm{~g}$, Massa Medicata Fermentata (Toasted Shen Qu) 15g/ 126g, Poria cocos (Fu Ling Pi) $30 \mathrm{~g} / 126 \mathrm{~g}$ and Radixet Rhizonma Ephedrae (Ma Huang Gen) 6g/ 126g. (Table 1). Some studies have revealed that Os Draconis and Concha Ostreae, key components of Longmu Tang, exerted sedation-like and antipruritic effects in treatment of AD. In addition, organic calcium in Os Draconis and Concha Ostreae could alleviate allergic symptoms ${ }^{[13]}$. Forsythia suspensa inhibited Staphylococcus aureus on the skin surface of patients with atopic dermatitis ${ }^{[14,15]}$. Poria cocos bark extract had potential as an oral immune suppressor for the treatment of atopic dermatitis through the generation of regulatory $T$ cells ${ }^{[16]}$. In previous trial, we confirmed that Longmu Tang granule could inhibit skin lesions and attenuate atopic itch through suppression of inflammation in atopic dermatitis. We intend to conduct a high-quality, well-designed, randomized, controlled, double-blind clinical trial to confirm the efficacy and safety of Longmu Tang granule for the treatment of patients with mild-to-moderate AD.

Table 1 Components of Longmu Tang granule

\begin{tabular}{|lll|}
\hline Chinese name & Latin name & English name \\
\hline Long Gu & Os Draconis & Fossilizid \\
\hline Calcined Mu Li & Concha Ostreae & Calcined concha ostreae \\
\hline Lian Qiao & Forsythia suspensa & Fructus forsythiae \\
\hline Toasted Shen Qu & Massa Medicata Fermentata & Toasted medicated leaven \\
\hline Fu Ling Pi & Poria cocos & Tuckahoe peel \\
\hline Ma Huang Gen & Radixet Rhizonma Ephedrae & Radix ephedrae \\
\hline
\end{tabular}




\section{Objectives $\{7\}$}

The aim of this RCT is to evaluate the effects and safety of Longmu Tang granule for the treatment of atopic dermatitis. This study hypothesizes that Longmu Tang granule can improve the clinical symptoms, quality of life and cognitive ability of patients with mild or moderate atopic dermatitis by attenuating inflammation, compared to that achieved using placebo treatment.

\section{Trial design $\{8\}$}

This is a single centre, randomized, double-blind, placebo-controlled clinical trial. The aim of this study is to assess the safety and effectiveness of Longmu Tang granule in treating atopic dermatitis with dampheat disturbing heart-mind.

\section{Methods: Participants, Interventions And Outcomes}

\section{Study setting $\{9\}$}

This trial will be conducted at Xiyuan Hospital of China Academy of Chinese Medical Sciences. A total of 60 patients will be recruited. All patients will be informed about the objectives, approaches, potential adverse effects, and advantages of this trial. After obtaining written informed consent from participants and their legal guardian, participants who meet the eligibility criteria will be randomly assigned to the treatment or placebo group in a 1:1 ratio. Participants will undergo an 8-week treatment period and an 8week follow up. The trial was registered at Chinese Clinical Trial Registry (ChiCTR).org ID: ChiCTR2100041591..

\section{Eligibility criteria $\{10\}$}

\section{Diagnostic criteria}

A diagnosis of AD will be established according to Hanifin and Rajka diagnostic criteria. Major criteria include four clinical symptoms (such as pruritus and typical morphology and distribution). Minor criteria include 32 clinical symptoms (such as xerosis, ichthyosis, palmar hyperlinearity, or keratosis pilaris). Participants will be diagnosed with AD patient if they meet three or more major criteria and three or more minor criteria at the same time.

\section{Inclusion criteria}

1. Clinical diagnosis of $A D$

2. Clinical diagnosis for TCM syndromes of damp-heat disturbing heart-mind

3. SCORAD score between 0 and 50

4. Aged 6-12 years old

5. Those who volunteered to participate in clinical trial observation and signed the informed consent form 
6. Compliance with treatment completion and follow-up

7. No intake of concomitant drugs during the observation period, except for those specified

\section{Exclusion criteria}

Participants with any of the following conditions will be excluded:

1. Received antihistamine drugs, topical corticosteroids or calcineurin inhibitors in the preceding 1 week

2. Presence of abnormal liver and kidney function or severe systemic diseases

3. Combined with other skin diseases and bacterial (fungal) infection that could interfere with the assessment of severity of $A D$

4. Patients with poor compliance with TCM treatment

5. Allergy to the ingredients in Longmu Tang granule

6. Currently participating in another clinical trial

\section{Who will take informed consent? $\{26 a\}$}

The researchers will design the questions, recruit patients, and conduct the study. The participants and his/her guardians will take the informed consent form if they would like to participate in the study. The informed consent form will cover the purpose, procedure, and potential benefits and risks of the trial.

\section{Additional consent provisions for collection and use of participant data and biological specimens \{26b\}}

Prior to obtaining the informed consent, we will confirm with the participants about the right to use their data. Participants' data will be shared with the other relevant authorities. The trial does not involve collecting and using biological specimens.

\section{Interventions}

\section{Explanation for the choice of comparators $\{6 \mathrm{~b}\}$}

The Longmu Tang granule placebo was selected as the comparator to access the effects and safety of Longmu Tang granule and minimize bias.

\section{Intervention description \{11a\}}

In the Longmu Tang group, participants will be allocated to receive Longmu Tang granule $(9.8 \mathrm{~g} / \mathrm{sachet})$ orally with $50 \mathrm{ml}$ of warm water twice a day for 8 weeks. Active granules, each containing $9.8 \mathrm{~g}$ of Longmu Tang, are equivalent to $126 \mathrm{~g}$ of crude medicine. Placebo granule is composed of $5 \%$ crude Longmu Tang and 95\% starch and is similar appearance and smell to Longmu Tang granule. Patients in the control group will take orally the placebo at the same dose and schedule as those followed by patients in the Longmu Tang group. Both Longmu Tang and placebo granules will be provided by Beijing Tcmages Pharmaceutical Co., LTD, with similar labels and packaging. According to the analysis, the 
quality of Longmu Tang granule meets the Chinese Medicine Standards of the State Food and Drug Administration (SFDA).

\section{Criteria for discontinuing or modifying allocated interventions $\{11 \mathrm{~b}\}$}

Participants who fail to complete the study due to various reasons or use prohibited drugs in the protocol, regardless of time or reason, will be considered as dropouts. Serious adverse events will be reported in accordance with the reporting requirements. If $A D$ worsen during study, the test should be terminated based on the decision from physician. The last recorded data for these participants will be included in data analyses. Data obtained from participants who use prohibited drugs in the protocol will be treated as invalid.

\section{Strategies to improve adherence to interventions $\{11 \mathrm{c}\}$}

The drug date and quantity of drug distribution and return will be recorded. Participants are required to return both the finished drug package and the unused drug. Furthermore, the health education and free consultations on the daily care of the participants will address the issue of adherence.

\section{Relevant concomitant care permitted or prohibited during the trial $\{11 \mathrm{~d}\}$}

Participants will be discouraged from receiving any other treatment or medication during the study period to prevent any effect on the results. In the course of treatment, if there is a lot of exudate or severe itching, the patient can moisten with the skin Kang lotion (Beijing Huayang Kuilong Pharmaceutical Co., Ltd., specification $50 \mathrm{ml} /$ bottle, national medicine Zhunzi Z19990045) 2-4 times a day. After being diluted 6 times with boiling water of $4^{\circ}-10^{\circ}, 8$ layers of medical gauze can be dipped into the lotion, gently wrung out, and then applied to the affected skin. Loratadine tablets (Caretan, Bayer Medicine (Shanghai) Co., Ltd., specification $10 \mathrm{mg} \times 6$ tablets, national medicine quasi-word H10970410) can also be taken orally once a day, $10 \mathrm{mg}$ each time, if the itching is difficult to resist. Details of the prescriptions and medications used will be strictly recorded in the case report form. The proportion of participants using rescue drugs in the groups will be compared. Other traditional Chinese medicines should be forbidden.

\section{Provisions for post-trial care $\{30\}$}

If an adverse event occurs in the clinical trial, the committee of medical experts will determine whether it is related to the Longmu Tang granule. Xiyuan Hospital of China Academy of Chinese Medical Sciences will provide the cost of treatment and corresponding economic compensation for the damage related to the trial.

\section{Outcomes $\{12\}$}

\section{Primary outcome}

The primary outcome of the trial is the effectiveness of Longmu Tang granule in improving the clinical symptoms of patients after 8-week treatment. The main evaluation of the primary outcome is 


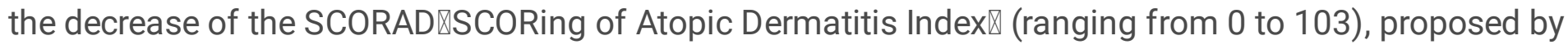
the European Task Force on AD (ETFAD). SCORAD includes the range of objective physical skin lesions (A), the severity of skin lesions (B), and the subjective symptoms of pruritus and the effect on sleep (C). This system is the internationally recognized gold standard for AD scoring. Higher scores can indicate more severe symptoms. The SCORAD before and after the treatment will be statistically analysed.

\section{Criteria for clinical efficacy}

Cure: asymptomatic, reduction of SCORAD before and after the treatment by $100 \%$.

Effective: symptoms were significantly relieved, and the reduction of SCORAD before and after the treatment by $\geq 50 \%$.

Ineffective: symptom remission is not obvious or no remission, and reduction of SCORAD before and after the treatment by $<50 \%$.

\section{Secondary outcomes}

1. The change of the Children's Dermatology Life Quality Index (CDLQI) score (from baseline to week 8): The DLQI is the most commonly used life index score of dermatological quality. This study will recruit children aged 6 to 12 years. Therefore, the Children's Dermatology Quality of Life Index (CDLQI) score is adopted.

2. The Number Cancel Test score (NCT) (from baseline to week 8): NCT is a traditional method to evaluate attention function. Attention function will be measured before and after the treatment, respectively, for 3 minutes each time, and includes the cancellation net score and cancellation error rate, comparing the number of correct strokes before and after the treatment (the greater the better) and the number of missed strokes and misstrokes (the less the better). Coarse score $=$ the sum of all correct marks, net score $=$ coarse score-(number of misstrokes + number of $1 / 2$ omissions), error rate $=$ (number of misstrokes + number of $1 / 2$ omissions) / coarse scores $\times 100 \%$.

3. The participants' expectations of Longmu Tang granule will be assessed at baseline using the following two questions: "Do you think Longmu Tang granule will be effective for treating the illness?" and "Do you think Longmu Tang granule will be effective for relieving the related symptoms of AD?" The response options will be "yes," "no," or "unclear."

4. Integral TCM syndrome score (from baseline to weeks 8 and 12; the scores and details are presented in Tables 2 and 3). A scale of 0-6 points will be used to score the patients with $A D$ according to the severity of clinical symptoms.

Tables 2 Traditional Chinese medicine syndrome score 


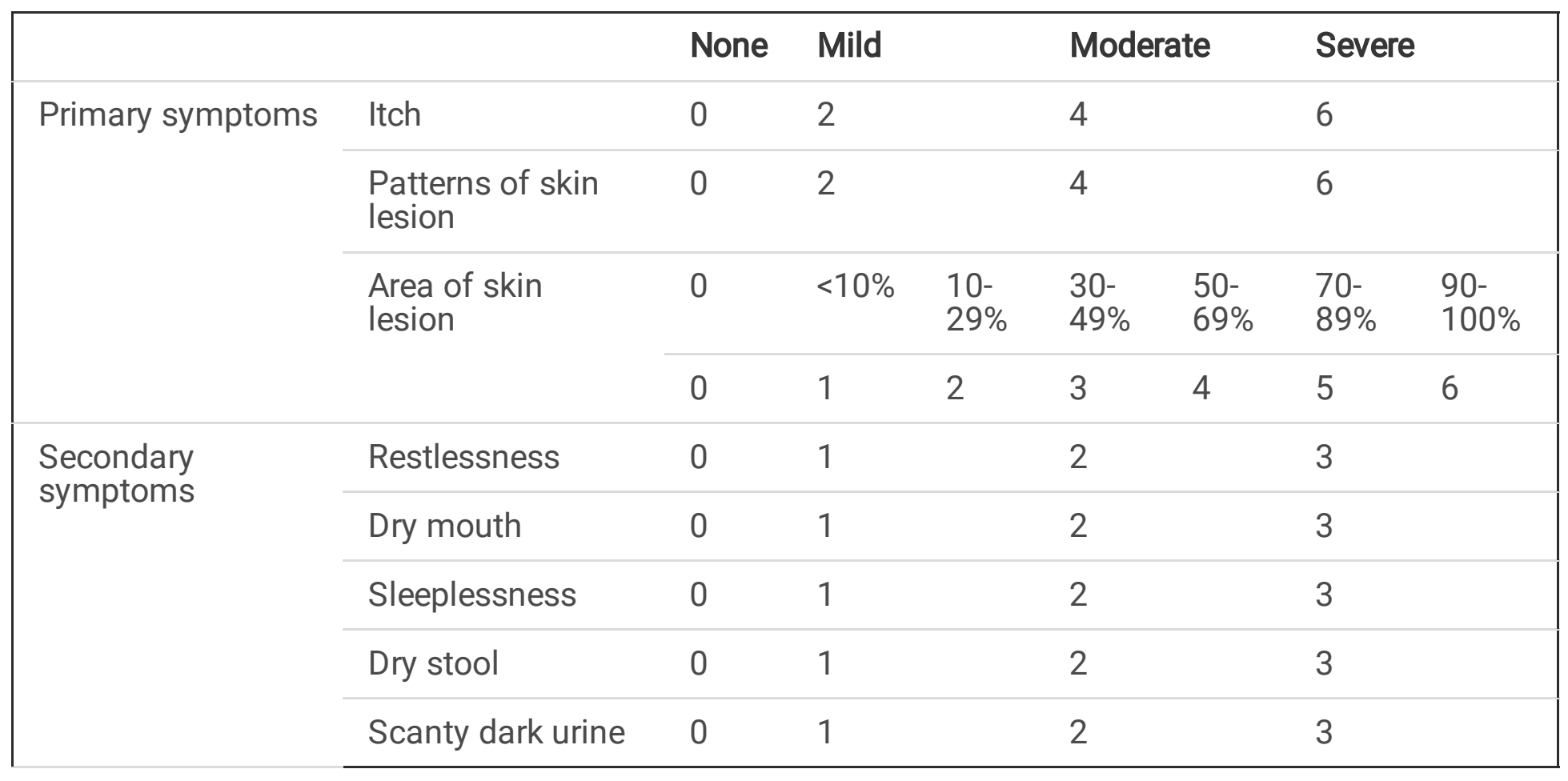

- Significant effect: inhibition or complete suppression of primary and secondary clinical symptoms and reduction of syndrome score by $\geq 95 \%$.

- Effective: significant improvement in clinical symptoms, and reduction of syndrome score by 70$95 \%$.

- Ineffective: with improvement in clinical symptoms, and reduction of syndrome score by $50-70 \%$.

- Invalid: reduction of treatment syndrome score by $<50 \%$.

Table 3 Detailed description of symptoms according to Traditional Chinese medicine 


\begin{tabular}{|c|c|c|c|c|}
\hline & None & Mild & Moderate & Severe \\
\hline Itch & $\begin{array}{l}\text { No } \\
\text { symptom }\end{array}$ & $\begin{array}{l}\text { Occasionally } \\
\text { occurrence and no } \\
\text { influence on study } \\
\text { and life }\end{array}$ & $\begin{array}{l}\text { Regular occurrence } \\
\text { and have influence } \\
\text { on study and life }\end{array}$ & $\begin{array}{l}\text { Frequent occurrence } \\
\text { and have serious } \\
\text { influence on study and } \\
\text { life }\end{array}$ \\
\hline $\begin{array}{l}\text { Patterns of } \\
\text { skin lesion }\end{array}$ & $\begin{array}{l}\text { No } \\
\text { symptom }\end{array}$ & $\begin{array}{l}\text { Erythema, papules, } \\
\text { or blisters }\end{array}$ & Oozing & $\begin{array}{l}\text { Skin thickening } \\
\text { (lichenification) }\end{array}$ \\
\hline $\begin{array}{l}\text { Area of skin } \\
\text { lesion }\end{array}$ & Calculated & ased on PASI score & & \\
\hline Restlessness & $\begin{array}{l}\text { No } \\
\text { symptom }\end{array}$ & Slight & Heavy & Heavy; irritable \\
\hline Dry mouth & $\begin{array}{l}\text { No } \\
\text { symptom }\end{array}$ & $\begin{array}{l}\text { Slight; no need to } \\
\text { drink water }\end{array}$ & $\begin{array}{l}\text { Severe; need to } \\
\text { drink water } \\
\text { occasionally }\end{array}$ & $\begin{array}{l}\text { Intolerable; need to } \\
\text { drink water frequently }\end{array}$ \\
\hline Sleeplessness & $\begin{array}{l}\text { No } \\
\text { symptom }\end{array}$ & $\begin{array}{l}\text { Slower falling } \\
\text { asleep or occasional } \\
\text { waking }\end{array}$ & $\begin{array}{l}\text { Difficult to fall } \\
\text { asleep and wake } \\
\text { up easily }\end{array}$ & Hardly able to sleep \\
\hline Dry stool & $\begin{array}{l}\text { No } \\
\text { symptom }\end{array}$ & $\begin{array}{l}\text { Dry stool; } 1 \text { time per } \\
\text { day }\end{array}$ & $\begin{array}{l}\text { Dry stool, } 1 \text { time in } \\
2-3 \text { days }\end{array}$ & $\begin{array}{l}\text { Difficult stool } \nabla>3 \text { days } \\
\text { each time }\end{array}$ \\
\hline $\begin{array}{l}\text { Scanty dark } \\
\text { urine }\end{array}$ & $\begin{array}{l}\text { No } \\
\text { symptom }\end{array}$ & $\begin{array}{l}\text { Urine volume is fine, } \\
\text { slightly yellowish in } \\
\text { color }\end{array}$ & $\begin{array}{l}\text { Small amount of } \\
\text { urine, yellow color, } \\
\text { slightly hot }\end{array}$ & Scanty dark urine \\
\hline
\end{tabular}

Abbreviations $\llbracket \mathrm{PASI}$, Psoriasis Area Severity Index

\section{Exploratory outcomes}


Serum inflammatory cytokines, such as serum Ig E, tumor necrosis factor (TNF) -a, interleukin-1 (IL-1) and interleukin-6 (IL-6), will be detected using enzyme-linked immunosorbent assay (ELISA) techniques at baseline and week 8 . The kits are from Sigma-Aldrich.

\section{Safety measures}

Safety measures include laboratory indexes (routine blood and urine analyses, and kidney and liver function tests) and adverse events. All adverse events will be tracked from the randomization to up to 16 weeks after the end of the intervention.

\section{Safety assessment}

Full blood count and urine, kidney and liver function tests will be performed before and after treatment. All details of severe adverse events (SAEs) and adverse events (AEs) will be recorded on the case report form (CRF). Any serious adverse events will be reported to the Ethics Approval Committee of Xiyuan Hospital of China Academy of Chinese Medical Sciences within 24 hours. Safety will be monitored at every visit for up to 8 weeks after the end of the intervention.

\section{Participant timeline $\{13\}$}

The visit schedule for all assessments (baselines, primary outcome, secondary outcomes, exploration outcomes and security indicators) is shown in Figure 1. At each assessment timepoint, the same investigator will evaluate the participants. During the treatment period (i.e., from baseline to 8 weeks), all combined therapeutic drugs will be recorded, including the name, dose and course of these drugs. The participants' expectations of Longmu Tang granule will be assessed at baseline. The primary outcome and the CDLQI score will be collected at the baseline, the $1^{\text {st }}, 2^{\text {nd }}, 4^{\text {th }}, 6^{\text {th }}$, and last visit. Exploratory outcomes, the NCT score and safety indicators will only be collected at the baseline and at the last visit. Table 4 summarises the schedule of screening, enrolment, intervention, assessments, and data collection.

Table 4 Schedule of enrolment, interventions, and assessments 


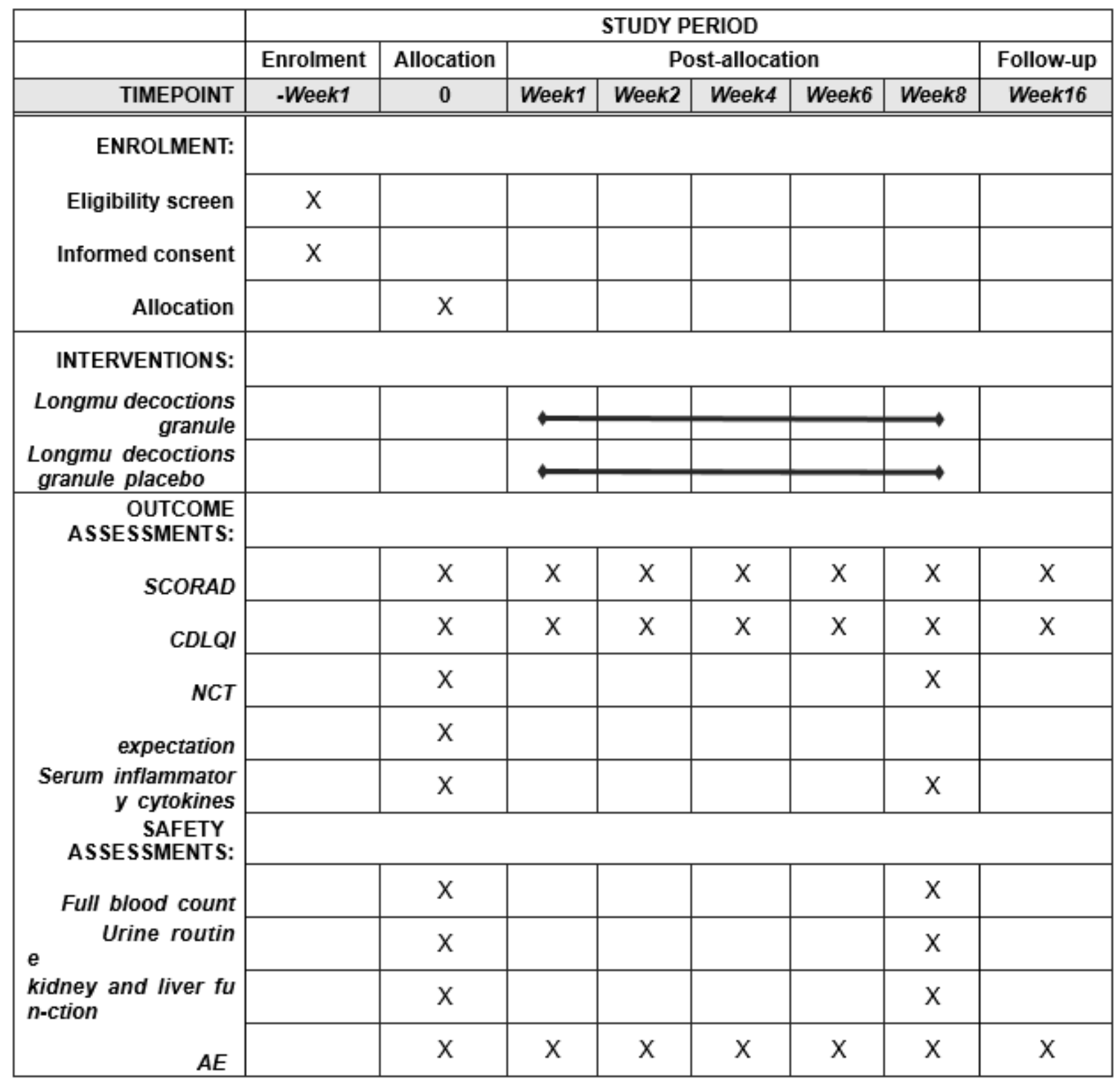

Abbreviations: SCORAD, the SCORing of Atopic Dermatitis; CDLQI, the Children's Dermatology Quality of Life Index; NCT, the Number Cancellation Test.

\section{Sample size $\{14\}$}

The sample size was calculated based on the expected value of efficacy, according to the previous exploratory clinical trial results and data analysis. The total efficacy rates in the study and control groups were $70 \%$ and $20 \%$, respectively. Assuming an alpha risk of $5 \%$ and a beta risk of $28 \%$, using the GPOWER software (V3.1.9.7), a sample size of 48 (24 participants in each group) was calculated. Considering a $20 \%$ loss to follow-up, this trial will require at least 60 participants (30 participants in each group) in the current study.

Recruitment \{15\} 
Sixty participants will be recruited from March 2021 to March 2022 at Xiyuan Hospital of China Academy of Chinese Medical Sciences via Internet advertisements (using WeChat) and posters in the hospital areas (such as the outpatient hall). The dermatologists will also ask the patients if they would like to participate in this trial during the treatment process of AD. A dermatologist will be responsible for screening potential participants and assessing whether they meet the eligibility criteria. A research assistant will provide the participant and his/her guardian with the informed consent form and interpret it for them in detail. The informed consent form will cover the purpose, procedure, and potential benefits and risks of the trial. The research assistant will perform a baseline assessment of all participants $(-1$ week to 0 week) prior to randomization. The demographic and clinical characteristics of the participants at baseline will be recorded on the case report form (CRF): age, sex, race, birthplace, body mass index (in $\mathrm{kg} / \mathrm{m}^{2}$ ), current address, history of allergic diseases, family history, severity of $A D$ assessed by Scoring Atopic Dermatitis (SCORAD) index (mild [0-24 score], moderate [25-50 score], severe [51-103 score]), quality of life assessed by the Children's Dermatology Life Quality Index (CDLQI) score, cognitive ability assessed by the Number Cancel Test (NCT), and safety evaluation assessed by laboratory index (full blood count, urine, kidney and liver function tests). An outline of procedures is illustrated in Figure. 1

\section{Assignment of interventions: allocation}

\section{Sequence generation $\{16 a\}$}

The random code will be generated by a statistician from the GCP Centre of Xiyuan Hospital of China Academy of Chinese Medical Sciences. Participants will be randomly assigned to either the treatment group or the placebo group, with a ratio of 1:1 and a fixed block of 4 .

\section{Concealment mechanism $\{16 \mathrm{~b}\}$}

The number of the randomization sequences and information about group allocation will be placed in ordered envelopes and subsequently sealed to ensure randomization concealment. The drugs will be labelled with random code on the small and large packages by GCP specialists of Xiyuan Hospital of China Academy of Chinese Medical Sciences.

\section{Implementation $\{16 \mathrm{c}\}$}

All participants and researchers will be unaware of the assignment until study completion. The same blinding code generated by statisticians will be provided to the research institution, and will not be broken during the trial period, except in the case of a serious adverse event that requires urgent breaking of the blinding code.

\section{Assignment of interventions: Blinding}

\section{Who will be blinded $\{17 \mathrm{a}\}$}


All participants and researchers will be blinded until the trial is completed.

\section{Procedure for unblinding if needed $\{17 \mathrm{~b}\}$}

For emergency blinding, each case corresponds to an emergency envelope with information about the coding and groups. Once any serious event occurs and patients need to be treated, the project leader will decide to initiate unblinding and open the corresponding emergency envelope. The case is then treated as a shedded case.

\section{Data collection and management}

\section{Plans for assessment and collection of outcomes $\{18 \mathrm{a}\}$}

The assessment and collection of outcomes are described as "outcomes," and data will be recorded in case report form (CRF) at each visit which can be found with the corresponding author.

\section{Plans to promote participant retention and complete follow-up $\{18 \mathrm{~b}\}$}

To maximize compliance and retention throughout the study, researchers will ensure that the study schedule and participation requirements are fully explained before the consent is obtained, and that participants are aware of the potential risks and benefits of treatment. Each participant will be provided with a copy of the signed informed consent form, containing the contact information of the investigators, so that they can contact the primary investigators if required. Moreover, all participants will receive phone calls before each visit to remind them of the time. Participants will receive free examinations and the study drug.

\section{Data management $\{19\}$}

The data will be imported into the Epidata software by two independent researchers. All researchers will receive professional training before and during this trial, including correct assessments, procedures regarding the use of the study drug, CRF completion and use of the Epidata. Auditors from Capital's Funds for Health Improvement and Research will supervise all trial-related procedures and data management.

\section{Confidentiality \{27\}}

All participants' data will be collected in specific reception room instead of public areas to avoid revealing their personal information to others. Initials will be used on the CRF and all documents will be stored confidentiality, accessible only to members of this study. Personal information about potential and enrolled participants will only be consulted by researchers of this study and will not be shared.

\section{Plans for collection, laboratory evaluation and storage of biological specimens for genetic or molecular analysis in this trial/future use $\{33\}$}

Not applicable, this trial does not involve biological specimens collecting. 


\section{Statistical methods}

\section{Statistical methods for primary and secondary outcomes $\{20 \mathrm{a}\}$}

Data analysis will be completed using the Statistical Packages of Social Sciences software (version 22.0), according to the per-protocol principle and the intention-to-treat principle. Normally distributed continuous variables will be reported as the mean \pm standard deviation; nonnormally distributed continuous variables will be reported as the median (interquartile range). Two independent sample t tests, the Wilcoxon test or paired $t$ test, will be applied for continuous variables, and the chi-square test and Fisher's exact probability method will be applied for categorical variables. The two-sided test will be uniformly used in the hypothesis test, with $P$ values $<0.05$ will be considered indicative of statistically significant and $P$ values $<0.01$ considered highly statistically significant.

\section{Interim analyses $\{21 \mathrm{~b}\}$}

Not applicable, the trial will be terminated when the sample reached 60 .

\section{Methods for additional analyses (e.g. subgroup analyses) \{20b\}}

Subgroup analyses will be performed by gender in future.

\section{Methods in analysis to handle protocol non-adherence and any statistical methods to handle missing data $\{20 \mathrm{c}\}$}

Participants with poor adherence, compliance of experimental drug $<80 \%$, or those no longer receiving medication and undergoing testing during the study will be included in the intention-to-treat analysis and excluded from the per-protocol analysis. A multiple data imputation procedure will be used to imput the missing data.

\section{Plans to give access to the full protocol, participant level-data and statistical code $\{31 \mathrm{c}\}$}

The full protocol, participant level-data, and statistical code are available from the corresponding author on reasonable request.

\section{Oversight and monitoring}

\section{Composition of the coordinating centre and trial steering committee $\{5 \mathrm{~d}\}$}

The coordinating centre is the department of dermatology of Xiyuan Hospital of China Academy of Chinese Medical Sciences, which will help to recruit participants and obtain consent. The trial steering committee is the Capital's Funds for Health Improvement and Research, which will supervise the trial and review the trial progress. 
Not applicable, as this is a low-risk intervention.

\section{Adverse event reporting and harms $\{22\}$}

All details of severe adverse events (SAEs) and adverse events (AEs) will be recorded on the case report form (CRF) and reported to the primary researcher, including the symptoms and severity, timing, action taken and outcomes. Any serious adverse events will be reported to the Ethics Approval Committee of Xiyuan Hospital of China Academy of Chinese Medical Sciences within 24 hours. If an adverse event occurs in the clinical trial, the committee of medical experts will determine whether it is related to the experimental drug and further decide whether to terminate the treatment based on the patient's condition. Other unintended effects of study interventions and study conduct will also be recorded and reported.

\section{Frequency and plans for auditing trial conduct $\{23\}$}

The Capital's Funds for Health Improvement and Research and the Ethics Approval Committee of Xiyuan Hospital of China Academy of Chinese Medical Sciences will meet once a year to review trial conduct.

\section{Plans for communicating important protocol amendments to relevant parties (e.g. trial participants, ethical committees) $\{25\}$}

We will notify the Ethics Approval Committee of Xiyuan Hospital of China Academy of Chinese Medical Sciences and the Capital's Funds for Health Improvement and Research when there are important changes in the study process such as the change of the sample size.

\section{Dissemination plans $\{31$ a $\}$}

The final results will be submitted to the Capital's Funds for Health Improvement and Research in the form of a research report. Trial results will be disseminated via peer-reviewed publications and scientific conferences /meetings.

\section{Discussion}

Atopic dermatitis is the complex chronic inflammatory skin disease, involving the critical components of epidermal barrier disruption, dysregulation of the immune response and genetic predisposition. Atopic dermatitis-related itch and frequent scratching behaviour have a substantial impairment to the patient's quality of life in health-related aspects such as sleep, physical activity, psychosocial and mental functioning. Roth et al. ${ }^{[17]}$ found that children with $A D$ were more likely to suffer from $A D H D$ than children without $A D$, and were more likely to show attention deficits in particular. Thus, the optimal treatment regimen for atopic dermatitis should include anti-inflammation, improvement of pruritus and attenuation of skin lesions.

The efficacy and advantages of TCM in treating atopic dermatitis have gradually been recognized. TCM including herbal formulae and bioactive ingredients exerts Traditional Chinese medicine (TCM) including 
herbal formulae and bioactive ingredients used to treat atopic dermatitis in attenuating skin lesion, preventing recurrence and reducing itch ${ }^{[18]}$. Traditional Chinese medicine (TCM) including herbal formulae and bioactive ingredients exerts pharmacological effects on atopic dermatitis. It has been a long history of TCM being used to treat atopic dermatitis, especially in preventing disease recurrence, maintaining long-term remission, and reducing disease burden.

However, much of the obtained outcomes from clinical trials of TCM herbs are not accepted due to the lack of strictly protocol in related fields. To accomplish our trial goal, this clinical trial design and conduct will comply with rigorous methodology and high-quality control. Longmu Tang granule is also produced with the standardized procedure according to Good Manufacturing Practice (GMP) protocol, which guarantees homogeneous active components and makes outcomes more credible. Currently, there are few TCM treatments involving syndrome differentiation therapy to patients with mild-to-moderate AD patients. To our knowledge, this is the first clinical trial that explore the efficacy and safety of a Longmu Tang granule for treating atopic dermatitis associated with damp-heat disturbing heart-mind. Our results from a previous clinical study showed that Longmu Tang granule can improved the severity of symptoms in $A D$ patients with the effective rate of $70 \%{ }^{[19]}$. These results may confer to broad therapeutic benefits as a disease-syndrome differentiation therapy for atopic dermatitis and itch.

There are some limitations for this study. First, the study will be performed at a single centre, which may not extrapolate the results to other ethnic groups or regions. Second, the sample size of this study is also relatively small, which may lead to overestimation of the efficacy of Longmu Tang granule. In conclusion, this trial will provide high-quality evidence on the effectiveness and safety of Longmu Tang granule, which is available to guide clinical practice for AD.

\section{Trial status}

The protocol version number is V3.2 and the date is 14, May 2020. The recruitment has commenced in March 2021 and will end in March 2022.

\section{Abbreviations}

AD: Atopic dermatitis; TCM: traditional Chinese medicine; SCORAD: SCORing of Atopic Dermatitis Index; CDLQI: the children's Dermatology Life Quality Index; NTC: the Number Cancel Test; TNF: tumor necrosis factor; IL-1: interleukin-1; IL-6: interleukin-6; ADHD: attention-deficit/hyperactivity disorder; QoL: the quality of life; Th: T helper; ChiCTR: Chinese Clinical Trial Registry; SFDA: the Chinese Medicine Standards of the State Food and Drug Administration; ETFAD: the European Task Force on AD; DLQI: the Children's Dermatology Life Quality Index; PASI: Psoriasis Area Severity Index; ELISA: enzyme-linked immunosorbent assay; SAEs: severe adverse events; AEs: adverse events; CRF: the case report form; GMP: Good Manufacturing Practice. 


\section{Declarations}

Acknowledgements

None.

\section{Authors' contributions $\{31 \mathrm{~b}\}$}

NL and HYC contributed to study design. JXR, YQL, MXM, YCT, JJW, FYT and YYS were responsible for conduct the study and recruit the participants. JXR, YQL and QYW conceived and drafted this protocol, and NL revised it. JJW, FYT, YQL and MXM collected and recorded the statistical data. JXR, HYC and TTS were involved in analyzing statistical data. NL and HYC supervise the study. All authors have read and agreed with the final manuscript.

\section{Funding $\{4\}$}

This study was supported by the Capital's Funds for Health Improvement and Research (No.2020-2-417249), The funding body played no role in the design of the study, collection, analysis, and interpretation of data and in the writing of the manuscript.

\section{Availability of data and materials $\{29\}$}

The principal investigators will have access to the final trial database, which will be available on reasonable request. Participants' personal information will be confidentially before, during and after the trial.

\section{Ethics approval and consent to participate $\{24\}$}

The trial was approved by the Ethics Approval Committee of Xiyuan Hospital of China Academy of Chinese Medical Sciences (approval number: 202XLA031-2). Participants regarding the trial protocols will sign the informed consent forms at the baseline and their personal information will remain strictly confidential,

\section{Consent for publication $\{32\}$}

Not applicable.

\section{Competing interests $\{28\}$}

The authors declare that they have no competing interests.

\section{References}

1. Langan SM, Irvine AD, Weidinger S. Atopic dermatitis. Lancet. 2020;396(10247):345-360. 
2. Kim Y, Blomberg M, Rifas-Shiman SL, Camargo CA Jr, Gold DR, Thyssen JP, et al. Racial/Ethnic Differences in Incidence and Persistence of Childhood Atopic Dermatitis. J Invest Dermatol. 2019;139(4): 827-834.

3. Guo Y, Li P, Tang J, Han X, Zou X, Xu G, et al. Prevalence of Atopic Dermatitis in Chinese Children aged 1-7ys. Sci Rep. 2016;6:29751.

4. Davidson WF, Leung DYM, Beck LA, Berin CM, Boguniewicz M, Busse WW, et al. Report from the National Institute of Allergy and Infectious Diseases workshop on "Atopic dermatitis and the atopic march: Mechanisms and interventions". J Allergy Clin Immunol. 2019;143(3):894-913.

5. Strom MA, Fishbein AB, Paller AS, Silverberg JI. Association between atopic dermatitis and attention deficit hyperactivity disorder in U.S. children and adults. Br J Dermatol. 2016;175(5):920-929.

6. Cao H, Yan S, Gu C, Wang S, Ni L, Tao H, et al. Prevalence of attention-deficit/hyperactivity disorder symptoms and their associations with sleep schedules and sleep-related problems among preschoolers in mainland China. BMC Pediatr. 2018;18(1):70.

7. Catal F, Topal E, Soylu N, Ozel Ozcan O, Celiksoy MH, Babayiğit A, et al. Psychiatric disorders and symptoms severity in preschool children with atopic eczema. Allergol Immunopathol (Madr). 2016;44(2):120-124.

8. Beattie PE, Lewis-Jones MS. A comparative study of impairment of quality of life in children with skin disease and children with other chronic childhood diseases. Br J Dermatol. 2006;155(1):145151.

9. Reed B, Blaiss MS. The burden of atopic dermatitis. Allergy Asthma Proc. 2018;39(6): 406-410.

10. Feld M, Garcia R, Buddenkotte J, Katayama S, Lewis K, Muirhead G, et al. The pruritus- and TH2associated cytokine IL-31 promotes growth of sensory nerves. J Allergy Clin Immunol. 2016;138(2):500-508.e24.

11. Li S, Kuchta K, Tamaru N, Lin Y, Iwasaki S, Wang R, et al. Efficacy of a novel herbal multicomponent traditional Chinese medicine therapy approach in patients with atopic dermatitis. Forsch Komplementmed. 2013;20(3):189-196.

12. Sheehan MP, Rustin MH, Atherton DJ, Buckley C, Harris DW, Brostoff J, et al. Efficacy of traditional Chinese herbal therapy in adult atopic dermatitis. Lancet. 1992;340(8810):13-17.

13. Zhang H, Zhang L, Liu Y. Studies on chemical components and pharmacological activities of Os Draconis (Longgu) and Ostreae Concha. China journal of Chinese materia medica. 2011;36(13):1839-40. [article in Chinese]

14. Wang JH, Mao W, Wang XH, et al. Analysis of bacterial colonization in skin lesions and non-skin lesions of patients with Atopic dermatitis. Chinese journal of leprosy and skin diseases. 2011;27(11) :774-776 [article in Chinese]

15. Jiang B, Su Y, Cai YH. Experimental Study on Inhibitory Effects and Its Mechanism of Forsythia on Staphylococcus aureus. Genomics and applied biology. 2018,08:3401-3406. [article in Chinese]

16. Bae MJ, See HJ, Choi G, Kang CY, Shon DH, Shin HS. Regulatory T Cell Induced by Poria cocos Bark Exert Therapeutic Effects in Murine Models of Atopic Dermatitis and Food Allergy. Mediators 
Inflamm. 2016;2016:3472608.

17. Roth N, Beyreiss J, Schlenzka K, Beyer H. Coincidence of attention deficit disorder and atopic disorders in children: empirical findings and hypothetical background. J Abnorm Child Psychol. $1991 ; 19(1): 1-13$.

18. Yan F, Li F, Liu J, Ye S, Zhang Y, Jia J, et al. The formulae and biologically active ingredients of Chinese herbal medicines for the treatment of atopic dermatitis. Biomed Pharmacother. 2020;127:110142.

19. Tian XY. Clinical observation and mechanism of jian pi zhu yun prescription in the treatment of pediatric atopic dermatitis. Beijing University of Chinese Medicine,2019. [academic dissertation in Chinese]

\section{Figures}




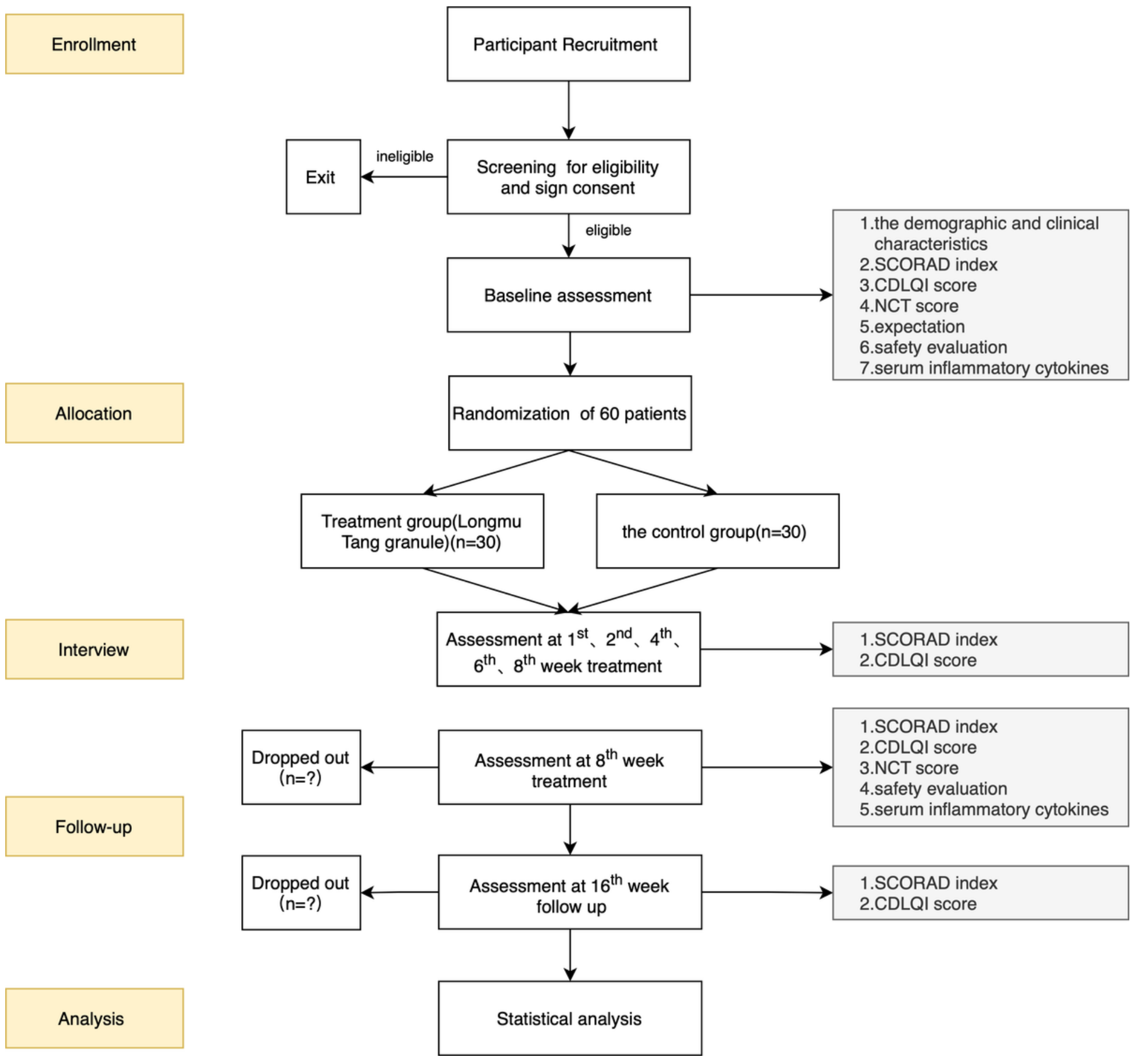

Figure 1

An outline of procedures 